\title{
Micromechanical Modeling of Creep Behavior in Particle-Reinforced Silicone-Rubber Composites
}

\section{Chao-Hsun Chen Associate Professor.}

\author{
Chaing-Ho Cheng \\ Former Graduate Student.
}

Institute of Applied Mechanics, National Taiwan University, Taipei 10764, Taiwan R.0.C.
A micromechanically based composite model is proposed to study the viscoelastic behavior of solid-filled rubber composites. A nonlinear So-Chen's (1991) mechanical model which describes the viscoelastic behavior of the rubber matrix is proposed to relate volume-average deformation and stress within the two-phase composite inclusion to the remote (macroscopic) fields. The influence of the volume fractions of inclusions on the overall creep strain of a rubber-matrix composite is investigated at the level of dilute concentration. The creep rate of the rubber matrix, which depends nonlinearly on the creep strain and the primary creep and secondary creep resulting from the viscous flow of creep deformation, is also considered in addition to the usual steady-state, or secondary, creep. The method developed for the calculation of the incremental process is based upon Eshelby's (1957) equivalence principle of an inhomogeneity-transformation problem and Mori-Tanaka's (1973) idea of mean-field stress. In order to examine the applicability of the model as well as the nonlinear stretch parameter, a series of experiments on solid-filled silicone rubbers has been carried out, which included constant rate of tensile tests and creep tests. It is demonstrated that this simple, albeit approximate micromechanical modeling is capable of predicting the volume fraction dependence of the time dependent creep, with characteristic consistency with the known elastic behavior.

\section{Introduction}

For nonlinear vicoelastic material, even under very small strain conditions, the stress-strain relationship is still nonlinear and the Boltzmann's superimposition method can no longer be applied. We can find suitable nonlinear elements to form the nonlinear mechanical model, such as So-Chen's (1991) nonlinear four-element Burger's (NFEB) model (Fig. 1), which can well describe the nonlinear mechanical behavior. They treated each individual molecular chain as the "entropy spring" and employed the rubber elasticity formula to replace the linear spring. Also, from the concept of the nonlinear activation energy jump over the energy barrier, they employed Eyring's activation energy dashpot to replace Newton's dashpot.

This paper is concerned with the quantitative determination of the time-dependent creep of a rubber-matrix composite, where solid particles are homogeneously dispersed in the rubber matrix. The particles are assumed to remain elastic, but at elevated temperature the rubber matrix may undergo both primary (transient) and the secondary (steady-state) creep. The creep rate of rubbers usually depends nonlinearly on the stress. The matrix and particles will be taken to be perfectly bonded together, without any void nucleation or growth. Here the composite as a whole is isotropic, and its overall creep behavior will be affected by the volume function of the particles. When such a composite is subjected to a constant, external stress, the initial response is elastic, and its overall elastic strain can be determined from its effective moduli.

Contributed by the Applied Mechanics Division of The AMBRICAN SOCIETY of Mechanical EngineERs for publication in the ASME Journal of ApPLIED Mechanics.

Discussion on this paper should be addressed to the Technical Editor, Professor Lewis T. Wheeler, Department of Mechanical Engineering, University of Houston, Houston, TX 77204-4792, and will be accepted until four months after final publication of the paper itself in the ASME JourNaL of APPLIED MECHANICS.

Manuscript received by the ASME Applied Mechanics Division, Aug. 18, 1994; final revision, Aug. 31, 1995. Associate Technical Editor: R. Abeyaratne.
As the stress-strain relation of the solid-filled rubber composite is highly nonlinear, we have used the secant elastic moduli in our theoretical derivation to capture the elastic modulus of every loading status. The theory makes use of a linear comparison material, whose elastic moduli at every instant is chosen to coincide with the average secant moduli of the rubber matrix to reflect its nonlinear state. Following Eshelby's (1957) equivalent-inclusion principle and Mori-Tanaka's (1973) mean-field method, the composite is subsequently replaced by the comparison material filled with equivalent transformation strains. This approach allows one to find the average stress of the matrix in terms of the macroscopic stress, and then by appealing to the constitutive equation of the rubber matrix, the overall stressstrain relation of the two-phase system can be easily determined.

This paper is organized as follows; the constitutive equations of the rubber matrix will be briefly recapitulated first, and then the initial stress state of the rubber matrix, suitable for the calculation of the initial creep rate, will be derived. The micromechanical principles involved in assessing the stress variation of the matrix following an incremental creep will be established subsequently, and the overall creep will be presented at the end. This result will then be checked by comparison with results from a series of experiments for glassbead-reinforced silicone rubber specimens.

\section{Neo-Hookean Rubber Elasticity and the Associ- ated Secant Moduli}

Let $\lambda_{i}$ be the principal stretches of deformation associated with the Cauchy-Green deformation tensor so that, for a NeoHookean material characterized by a single constant $\mu$, the principal Cauchy stress components $\sigma_{k k}$ are determined by (Truesdell and Noll, 1965):

$$
\sigma_{k k}=\mu \lambda_{k}^{2}-p, \quad k=1,2,3,
$$




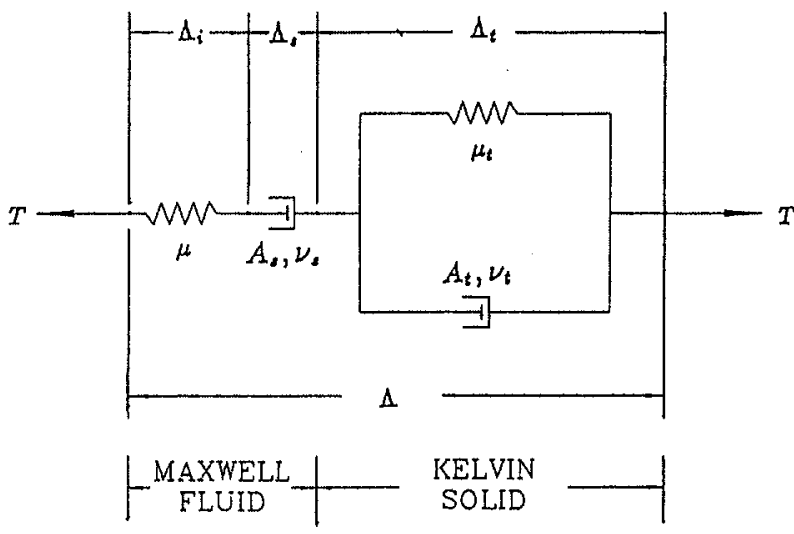

Fig. 1 So-Chen's nonlinear four-element burger's model

where $p$ is an unknown hydrostatic stress. The above equation is augmented by the constant volume constraint relation:

$$
\lambda_{1} \lambda_{2} \lambda_{3}=1 \text {. }
$$

Consider now the following generalized tensile loading:

$$
\sigma_{11}=P, \quad \sigma_{22}=\sigma_{33}=Q,
$$

where $Q$ vanishes for a simple tensile loading. It follows from (2.1) and (2.2) that the associated deformation is

$$
\begin{gathered}
\lambda_{1}=\lambda(R)=M(R)+\frac{R}{3 \mu M(R)} \\
\lambda_{2}=\lambda_{3}=\sqrt{\lambda}^{-1} \\
M(R)=\left\{\frac{1}{2}+\left[\frac{1}{4}-\left(\frac{R}{3 \mu}\right)^{3}\right]^{1 / 2}\right\}^{1 / 3} \\
R=P-Q
\end{gathered}
$$

and

$$
R=\mu\left(\lambda^{2}-\frac{1}{\lambda}\right)
$$

It is clear from the above that the desired material constant $\mu$ may be deduced by fitting (2.8) to an experimentally measured curve for the simple tension test

$$
R=P=\sigma_{11}=\sigma, \quad Q=0,
$$

recalling that the three principal engineering strains $\epsilon_{k k}$ are related to the principal stretches by $\epsilon_{k k}=\lambda_{k}-1$.

The generalized tensile loading (2.3) and the strains may be made to satisfy the linear elasticity relations. The secant shear modulus, $\mu^{s}$, and secant bulk modulus, $\kappa^{s}$, follow from the usual definitions. We have

$$
\begin{gathered}
\kappa^{s}=\frac{1}{3}\left(\frac{R+3 Q}{\lambda+2 \lambda^{-1 / 2}-3}\right) \\
\mu^{s}=\frac{1}{2}\left(\frac{R}{\lambda-\lambda^{-1 / 2}}\right) .
\end{gathered}
$$

For rubbers, nonlinear behavior exhibits even under small strains. The linear mechanical model can not be applied to such materials directly.

So-Chen's (1991) NFEB model is employed here to describe the nonlinear viscoelastic behavior of the elastomers. The total nonlinear stretch $\Lambda$ can be expressed as follows:

$$
\begin{aligned}
\Lambda(t) & =\frac{T}{\mu}+A_{s} \sinh \left(\nu_{s} T\right) t \\
+ & \frac{T}{\mu_{t}}\left\{1-\frac{2}{\nu_{t} T} \tan ^{-1}\left[\tanh \left(\frac{\nu_{t} T}{2}\right) \exp \left(-A_{t} \nu_{t} \mu_{t} t\right)\right]\right\}
\end{aligned}
$$

where

$$
\Lambda=\lambda-\frac{1}{\lambda^{2}}
$$

There are six material parameters, namely, $\mu, A_{s}, \nu_{s}, \mu_{t}, A_{t}$, and $\nu_{t}$, in the nonlinear model as well as in Eq. (2.12). In general, some experimental data from the two creep curves using the method of least squares are required to determine these six parameters. This equation will be proved correct by experimental results.

Equation (2.12) leads to the total nonlinear stretch rate, $\dot{\Lambda}$, as

$\dot{\Lambda}=A_{s} \sinh \left(\nu_{s} T\right)$

$$
+\frac{2 A_{t} \tanh \left(\frac{\nu_{t} T}{2}\right) \exp \left(-\nu_{t} \mu_{t} A_{t} t\right)}{1-\left[\tanh \left(\frac{\nu_{t} T}{2}\right) \exp \left(-\nu_{t} \mu_{t} A_{t} t\right)\right]^{2}} .
$$

Equation (2.13) can be recasted as

$$
\dot{\lambda}=\frac{\dot{\Lambda}}{1+\frac{2}{\lambda^{3}}} .
$$

At the initial stage, the rubber matrix satisfies the incompressibility constraint. When the creep behavior starts, the incompressible constraint no longer holds. As its difference is stil small compared to the total deformation, for convenience of computation, here, we approximate the relation between true stress, $\sigma$, and engineering stress, $T$, by using the incompressible constraint of rubber elasticity as

$$
T=\sigma \lambda_{2} \lambda_{3} \approx \frac{\sigma}{\lambda_{1}}=\frac{\sigma}{\lambda} .
$$

Substituting Eqs. (2.14) and (2.16) into Eq. (2.15) leads to the stretch rate, $\dot{\lambda}$, as

$$
\begin{aligned}
& \dot{\lambda}=\left\{A_{s} \sinh \left(\nu_{s} \frac{\sigma}{\lambda}\right)\right. \\
& \left.+\frac{2 A_{t} \tanh \left(\frac{\nu_{t} \sigma}{2 \lambda}\right) \exp \left(-\nu_{t} \mu_{t} A_{t} t\right)}{1-\left[\tanh \left(\frac{\nu_{t} \sigma}{2 \lambda}\right) \exp \left(-\nu_{t} \mu_{t} A_{t} t\right)\right]^{2}}\right\} /\left(1+\frac{2}{\lambda^{3}}\right) \\
& =F(\sigma, \lambda, t) .
\end{aligned}
$$

As the matrix phase in a composite system often exits in a triaxial stress state, we can generalize Eq. (2.17) to uniaxial systems for an isotropic and incompressible material by employing the plastic flow idea. Therefore, the effective stretch rate, $\dot{\lambda}_{e}$, can be generalized as a function of Von-Misses's effective stress, $\sigma_{e}$, effective stretch, $\lambda_{e}$, and time, $t$, as

$$
\dot{\lambda}_{e}=F\left(\sigma_{e}, \lambda_{e}, t\right) \text {. }
$$


The differentiation of Eq. (2.2) can be written as

$$
\frac{d \lambda_{1}}{\lambda_{1}}+\frac{d \lambda_{2}}{\lambda_{2}}+\frac{d \lambda_{3}}{\lambda_{3}}=0
$$

or

$$
d\left(\ln \lambda_{1}\right)+d\left(\ln \lambda_{2}\right)+d\left(\ln \lambda_{3}\right)=0 .
$$

For small deformation, Eq. (2.19a) can be reduced to the linear elasticity relation

$$
d \epsilon_{11}+d \epsilon_{22}+d \epsilon_{33}=0 .
$$

Equations (2.19a) and (2.20) show that for large deformation we can replace $\epsilon_{i}$ with $\ln \lambda_{i}$ and replace $\epsilon_{e}$ with $\ln \lambda_{e}$. By these analogous results, in the principle direction we have assumed the effective stress, $\sigma_{e}$, and effective stretch, $\lambda_{e}$, relation of large deformation is as follows:

$$
\begin{aligned}
\sigma_{e}=\frac{1}{\sqrt{2}}\left[\left(\sigma_{11}-\sigma_{22}\right)^{2}+\left(\sigma_{22}-\right.\right. & \left.\sigma_{33}\right)^{2} \\
& \left.+\left(\sigma_{33}-\sigma_{11}\right)^{2}\right]^{1 / 2} \\
\ln \lambda_{e}=\frac{\sqrt{2}}{3}\left[\left(\ln \lambda_{1}-\ln \lambda_{2}\right)^{2}\right. & +\left(\ln \lambda_{2}-\ln \lambda_{3}\right)^{2} \\
& \left.+\left(\ln \lambda_{3}-\ln \lambda_{1}\right)^{2}\right]^{1 / 2}
\end{aligned}
$$

Substituting Eqs. (2.21a) and (2.21b) into Eq. (2.18), we have the effective stretch rate $\dot{\lambda}_{e}$ relation. Following the PrandtlReuss flow rule idea, we have assumed that the flow rule of large deformation is

$$
d\left(\ln \lambda_{i}\right)=\frac{3}{2} \frac{d\left(\ln \lambda_{e}\right)}{\sigma_{c}^{\prime}} \sigma_{i i}^{\prime}, \quad i=1 \sim 3,
$$

where $\boldsymbol{\sigma}^{\prime}$ is the deviatoric stress. Equation (2.22) can be rewritten as

$$
d \epsilon_{i i}=d \lambda_{i}=\frac{3}{2} \frac{d \lambda_{e}}{\lambda_{e}} \frac{\lambda_{i}}{\sigma_{e}} \sigma_{i i}^{\prime}=\frac{3}{2} \frac{F\left(\lambda_{e}, \sigma_{e} t\right) \lambda_{i}}{\lambda_{e} \sigma_{e}} \sigma_{i i}^{\prime} d t
$$

or

$$
\dot{\epsilon}_{i i}=\frac{3}{2} \frac{F\left(\lambda_{e}, \sigma_{e}, t\right) \lambda_{i}}{\lambda_{e} \sigma_{e}} \sigma_{i i}^{\prime}
$$

\section{The Initial Stress of the Rubber Matrix}

Consider a representative volume of a glass bead-reinforced silicone rubber which is assumed to be statistically homogeneous and macroscopically isotropic. The silicone rubber is referred to as the matrix, and the volume fraction of the glass bead is denoted by $f_{1}$. Following the exposition of Tandon and Weng (1988) and Weng (1990), we introduce an identically shaped of comparison sample made of pure silicone rubber. Let the composite and the pure silicone rubber comparison sample both be subjected to the boundary traction, which will give rise to a uniform generalized tensile state

$$
\overline{\boldsymbol{\sigma}}=(\bar{P}, \bar{Q}), \quad \bar{R}=\bar{P}-\bar{Q} .
$$

The strain, $\boldsymbol{\epsilon}^{\circ}$, in the comparison sample is related to the above by

$$
\overline{\boldsymbol{\sigma}}=\mathbf{C}_{o}^{s} \boldsymbol{\epsilon}^{o},
$$

The average strain, $\boldsymbol{\epsilon}^{(0)}, \boldsymbol{\sigma}^{o}$ in the matrix of the composite cannot be the same as $\epsilon^{o}, \overline{\boldsymbol{\sigma}}$ and may be represented by

$$
\boldsymbol{\sigma}^{(o)}=\overline{\boldsymbol{\sigma}}+\tilde{\boldsymbol{\sigma}}=\mathbf{C}_{o}^{s} \boldsymbol{\epsilon}^{(o)}=\mathbf{C}_{o}^{s}\left(\boldsymbol{\epsilon}^{o}+\tilde{\boldsymbol{\epsilon}}\right) .
$$

The introduction of an equivalent transformation strain, $\epsilon^{*}$, combined with Mori-Tanaka's concept, leads to the identity

$$
\begin{aligned}
\boldsymbol{\sigma}^{(1)} & =\mathbf{C}_{1}\left(\boldsymbol{\epsilon}^{o}+\tilde{\boldsymbol{\epsilon}}+\boldsymbol{\epsilon}^{p}\right) \\
& =\mathbf{C}_{o}^{s}\left(\boldsymbol{\epsilon}^{o}+\tilde{\boldsymbol{\epsilon}}+\boldsymbol{\epsilon}^{p}-\boldsymbol{\epsilon}^{*}\right)
\end{aligned}
$$

where $\boldsymbol{\epsilon}^{p}$ and $\boldsymbol{\epsilon}^{*}$ are related by

$$
\boldsymbol{\epsilon}^{p}=\mathbf{S}_{o}^{s} \epsilon^{*}
$$

in which $\mathbf{S}_{o}^{s}$ is the Eshelby tensor (calculated in the elastic comparison material) associated with spherical inclusions and $\mathbf{C}_{o}^{s}$.

Finally, since $\overline{\boldsymbol{\sigma}}$ of (3.1) is also the uniform stress of the composite, we have

$$
\overline{\boldsymbol{\sigma}}=f_{1} \boldsymbol{\sigma}^{(1)}+\left(1-f_{1}\right) \boldsymbol{\sigma}^{(o)},
$$

where $f_{1}$ is the volume fraction of the glass bead. Following the above equations (3.3), (3.4), (3.5), and (3.6) we have

$$
\begin{gathered}
\boldsymbol{\epsilon}^{*}=\left(\mathbf{A}^{-1}-\left(1-f_{1}\right) \mathbf{S}^{s}-f_{1} \mathbf{I}\right)^{-1} \cdot \boldsymbol{\epsilon}^{o} \\
\mathbf{A}^{-1}=\mathbf{I}-\mathbf{C}_{o}^{s^{-1}} \mathbf{C}_{1} .
\end{gathered}
$$

The total strain of the composite is given by the weighted average of those strains of the matrix and inclusion, i.e.,

$$
\overline{\boldsymbol{\epsilon}}=\left(1-f_{1}\right) \boldsymbol{\epsilon}^{(0)}+f_{1} \boldsymbol{\epsilon}^{(1)}=f_{1} \boldsymbol{\epsilon}^{*}+\boldsymbol{\epsilon}^{o} .
$$

Substituting (3.7) into the above and using (3.2), we finally conclude that

$$
\overline{\boldsymbol{\sigma}}=\mathbf{C}^{s} \overline{\boldsymbol{\varepsilon}},
$$

where

$$
\mathbf{C}^{s}=C_{o}^{s}\left[\mathbf{I}+f_{1}\left(\mathbf{A}^{-1}-\left(1-f_{1}\right) \mathbf{S}^{s}-f_{1} \mathbf{I}\right)^{-1}\right]^{-1}
$$

and $\bar{C}^{s}$ is but the secant elasticity tensor for the composite at the stress level (3.1).

For convenience of measurement in the simple tension test, we need to use the relation between the engineering stress, $T$, and true stress, $\bar{\sigma}_{11}$, as

$$
T=\bar{\sigma}_{11}\left(1+\bar{\epsilon}_{33}\right)\left(1+\bar{\epsilon}_{22}\right) .
$$

The final product of this section is the composite stress-strain relations (3.11). The secant elasticity tensor, $\bar{C}^{s}$, however, depends on $\overline{\boldsymbol{\sigma}}$ via $\boldsymbol{\sigma}^{(\boldsymbol{})}$, the stress of the matrix of the composite. Thus, an iteration procedure must be improvised to facilitate the computation. We use the simpler hard-bead approximation to illustrate the procedure described as follows. Using (2.10) and (2.11), $\mu_{o}^{s}$ and $\kappa_{o}^{s}$ are derived; therefore, $C_{o}^{s}$ is obtained. Using (3.7), (3.9), and (3.11), $\bar{C}^{s}$ and $\vec{\epsilon}$ are derived, respectively. Finally, via (3.3), we have $\boldsymbol{\sigma}^{(o)}$. Then, we go back to (2.10) and (2.11) to check $\mathbf{C}_{o}^{s}$. By this iteration procedure, we can have the stress-strain solution of this composite system.

\section{The Micromechanics of Creep Behavior in Solid- Filled Rubber Composites}

As the stress-strain relation of the solid-filled rubber composites is highly nonlinear, to capture the elastic response for each time increment, we have used the secant moduli approach in our theoretical derivation. In Section 3 we made use of a linear comparison material, whose elastic moduli at every instant is chosen to coincide with the secant moduli of the rubber matrix to reflect its nonlinear state. Following Eshelby's (1957) equivalent-inclusion principle and Mori-Tanaka's (1973) mean stress by the comparison material filled with equivalent transformation strains, the secant moduli, $\mathbf{C}_{o}^{s}$, of different volume fractions of solid-filled rubber composites under any loading status can be expressed in terms of the functions of stress of the rubber matrix, $\sigma^{(o)}$. From Section 3, we have the stress distribution of the rubber matrix, $\sigma^{(o)}$, and the strain of the matrix, $\epsilon^{(o)}$, under a given stress, $\overline{\boldsymbol{\sigma}}$, individually, as 


$$
\begin{aligned}
\boldsymbol{\sigma}^{(o)}=\mathbf{C}_{o}^{s}\left(\mathbf{A}^{-1}-\mathbf{S}_{o}^{s}\right) & \\
& \times\left[\mathbf{A}^{-1}-\left(1-f_{1}\right) \mathbf{S}_{o}^{s}-f_{1} \mathbf{I}\right]^{-1} \mathbf{C}_{o}^{s^{-1}} \overline{\boldsymbol{\sigma}} \\
\boldsymbol{\epsilon}^{(o)}=\left(\mathbf{A}^{-1}-\right. & \left.\mathbf{S}_{o}^{s}\right) \\
& \left.\times\left[\mathbf{A}^{-1}-\left(1-f_{1}\right) \mathbf{S}_{o}^{s}-f_{1} \mathbf{I}\right)\right]^{-1} \mathbf{C}_{o}^{s^{-1}} \overline{\boldsymbol{\sigma}}, \\
& \mathbf{A}=\mathbf{I}-\mathbf{C}_{o}^{s^{-1}} \mathbf{C}_{1} .
\end{aligned}
$$

Substitution of Eqs. (4.1a) and (4.1b) into Eqs. (2.17) and (2.24) leads to the creep rate of the rubber matrix. Under a given $\bar{\sigma}$, the initial creep rate of the rubber matrix then can be calculated, and this, over a time increment $d t$, generates an incremental creep strain

$$
d \boldsymbol{\epsilon}^{c}=\dot{\boldsymbol{\epsilon}} d t
$$

Such is also the case for any subsequent time increment. Our concern now is the nature of the stress redistribution between the inclusions and rubber matrix following an incremental creep, with $\tilde{\sigma}$ kept constant.

In general, an incremental creep in the rubber matrix is also accompanied by a stress reduction, which has to be carried over by the elastic inclusions to maintain a constant $\overline{\boldsymbol{\sigma}}$. For a nonlinear matrix experiencing both primary and secondary creep, the determination of a precise solution for such a problem is not simple. Here we use the approximate method suggested previously by the writers Zhu and Weng (1987, 1989, 1990), which was originally formulated for the self-consistent determination of the time-dependent creep of polycrystals (Weng, 1981). This model takes the constraint power of the matrix to be elastic and, therefore, is more suitable for a small creep strain range. Since our matrix is rubber material, its stress-strain relation is highly nonlinear, so the secant moduli approach is employed here. Also, in our creep test, we can only fix a constant loading force instead of the constant loading stress, $\boldsymbol{\sigma}$. Due to the large deformation of the rubber matrix, the loading stress, $\overline{\boldsymbol{\sigma}}$, increases as the creep deformation increases. The stress increment, $d \overline{\boldsymbol{\sigma}}$, is due to the reduction of the cross-section area of the specimen. This stress increment doesn't cause the elastic strain to increase, but it does contribute to the stretch rate, $\dot{\lambda}$, of the next time-increment stage. Therefore, the stress increment, $d \overline{\boldsymbol{\sigma}}$, needs to be included for each time increment stage.

For solid-filled rubber composites, we have also employed the same idea, of that within the time interval $d t$, when there is a creep strain $d \epsilon^{c}$ of the rubber matrix then $d \bar{\epsilon}$ will also increase. As a result, the cross-section area $A$ will decrease, causing $d \boldsymbol{\sigma}$, in turn, to increase. Therefore, from $(4.1 a) d \boldsymbol{\sigma}^{(o)}$ will also increase. At the next time-step, the increase of $d \boldsymbol{\sigma}^{(0)}$ must be included in the calculation of $d \epsilon^{c}$.

To estimate the average stress variations in both phases, we first take the inclusions out and let the matrix creep homogeneously over a short time $d t$. The inclusions are now subjected to a boundary traction corresponding to a homogeneous stress, $\mathrm{C}_{1} d \epsilon^{c}$, so that they fit perfectly into the deformed holes.

A negative traction, $-\mathbf{C}_{1} d \epsilon^{c}$, is now applied to the bonded interfaces to remove the unwanted traction discontinuity. This is equivalent to having an Eshelby's "stress-free" strain,

$$
d \epsilon^{*}=-d \epsilon^{c},
$$

in the context of the inhomogeneity/transformation problem (see also Mura, 1987). Then, by means of Mori and Tanaka's (1973) concept of mean-field approximation, the average stress variation in the rubber matrix can be written as

$$
d \boldsymbol{\sigma}^{(o)}=d \tilde{\boldsymbol{\sigma}}=\mathbf{C}_{o}^{s} d \tilde{\boldsymbol{\epsilon}},
$$

in parallel with (3.3). Similarly, in parallel with (3.4), the mean-stress variation of the inclusions can be cast as

$$
\begin{aligned}
d \boldsymbol{\sigma}^{(1)} & =d \tilde{\boldsymbol{\sigma}}+d \boldsymbol{\sigma}^{p}=\mathbf{C}_{1}\left(d \tilde{\boldsymbol{\epsilon}}+d \boldsymbol{\epsilon}^{p t}-d \boldsymbol{\epsilon}^{*}\right), \\
& =\mathbf{C}_{o}^{s}\left(d \tilde{\boldsymbol{\epsilon}}+d \boldsymbol{\epsilon}^{p t}-d \boldsymbol{\epsilon}^{*}-d \boldsymbol{\epsilon}^{* *}\right),
\end{aligned}
$$

noting that a stress-free transformation strain, $d \epsilon^{*}$, already exists in the inclusion. Strain $d \epsilon^{* *}$ is Eshelby's equivalent transformation strain, and, together with $d \epsilon^{*}$, they form the total transformation strain, such that

$$
d \boldsymbol{\epsilon}^{p}=\mathbf{S}_{o}^{s}\left(d \boldsymbol{\epsilon}^{*}+d \boldsymbol{\epsilon}^{* *}\right) .
$$

The boundary condition $d \overline{\boldsymbol{\sigma}}=\Sigma f_{r} d \boldsymbol{\sigma}^{(r)}=0$ further leads to

$$
d \tilde{\boldsymbol{\sigma}}=-f_{1} d \boldsymbol{\sigma}^{p r},
$$

or

$$
\begin{aligned}
d \tilde{\boldsymbol{\epsilon}} & =-f_{1}\left[d \boldsymbol{\epsilon}^{p}-\left(d \boldsymbol{\epsilon}^{*}+d \boldsymbol{\epsilon}^{* *}\right)\right] \\
& =-f_{1}\left(\mathbf{S}_{o}^{s}-\mathbf{I}\right)\left(d \boldsymbol{\epsilon}^{*}+d \boldsymbol{\epsilon}^{* *}\right) .
\end{aligned}
$$

To find the overall strain variation over $d t$, we recall that a uniform creep strain, $d \epsilon^{c}$, took place before the inclusions were placed back into the deformed holes. It follows that

$$
\begin{aligned}
d \overline{\boldsymbol{\epsilon}}^{c} & =d \boldsymbol{\epsilon}^{c}+\left(1-f_{1}\right) d \tilde{\boldsymbol{\epsilon}}+f_{1}\left(d \tilde{\boldsymbol{\epsilon}}+d \boldsymbol{\epsilon}^{p}\right) \\
& =d \boldsymbol{\epsilon}^{c}+f_{1}\left(d \boldsymbol{\epsilon}^{*}+d \boldsymbol{\epsilon}^{* *}\right),
\end{aligned}
$$

where the strain increment, $d \epsilon^{c}$, is the inclusion's deformation after the inclusion is taken out. Meanwhile, the composite now has the uniform deformation strain, $d \epsilon^{c}$. Therefore, we need to add $d \boldsymbol{\epsilon}^{c}$ to form the total strain increment, $d \overline{\boldsymbol{\epsilon}}^{c}$. Using Eqs. (4.5), (4.6), and (4.8), d $\epsilon^{*}+d \epsilon^{* *}$ then can be derived as

$$
d \boldsymbol{\epsilon}^{*}+d \boldsymbol{\epsilon}^{* *}=\mathbf{B}_{1} d \boldsymbol{\epsilon}^{*}
$$

where

$$
\begin{gathered}
\mathbf{B}_{1}=\left(\mathbf{I}-\left(\mathbf{1}-f_{1}\right) \mathbf{A} \mathbf{S}_{o}^{s}-f_{1} \mathbf{A}\right)^{-1}(\mathbf{I}-\mathbf{A}) \\
\mathbf{A}=\left(\mathbf{I}-\mathbf{C}_{o}^{s^{-1}} \mathbf{C}_{1}\right) .
\end{gathered}
$$

Substituting Eqs. (4.3) and (4.10) into Eq. (4.9) leads to

$$
d \overline{\boldsymbol{\epsilon}}^{c}=\left(\mathbf{I}-f_{1} \mathbf{B}_{1}\right) d \boldsymbol{\epsilon}^{c} .
$$

Therefore, the stress increment of the matrix, $d \boldsymbol{\sigma}^{(0)}$, and the strain increment of the matrix, $d \epsilon^{(0)}$, then can be derived as

$$
\begin{gathered}
d \boldsymbol{\sigma}^{(o)}=f_{1} \mathbf{C}_{o}^{s}\left(\mathbf{S}_{o}^{s}-\mathbf{I}\right) \mathbf{B}_{1} d \boldsymbol{\epsilon}^{c} \\
d \boldsymbol{\epsilon}^{(o)}=f_{1}\left(\mathbf{S}_{o}^{s}-\mathbf{I}\right) \mathbf{B}_{1} d \boldsymbol{\epsilon}^{c}+d \boldsymbol{\epsilon}^{c} .
\end{gathered}
$$

The total creep strain of the composite system, $\overline{\boldsymbol{\epsilon}}$, and the rubber matrix, $\overline{\boldsymbol{\epsilon}}^{(o)}$, after a time increment can be written as

$$
\begin{gathered}
\boldsymbol{\epsilon}(t+d t)=\boldsymbol{\epsilon}(t)+d \boldsymbol{\epsilon}(t) \\
\boldsymbol{\epsilon}^{(o)}(t+d t)=\boldsymbol{\epsilon}^{(o)}(t)+d \boldsymbol{\epsilon}^{(o)}(t) .
\end{gathered}
$$

The stress increment, $d \bar{\sigma}$, due to the reduction of the crosssection area, is

$$
\begin{gathered}
d \bar{\sigma}_{11}=\frac{T}{\left(1+\bar{\epsilon}_{22}+d \bar{\epsilon}_{22}\right)^{2}}-\frac{T}{\left(1+\bar{\epsilon}_{22}\right)^{2}} \\
d \bar{\sigma}_{22}=0=d \bar{\sigma}_{33} .
\end{gathered}
$$

Due to the reduction of the cross-section area, from Eq. (4.1) we can get the stress increments of the rubber matrix as

$$
\begin{aligned}
d \overline{\boldsymbol{\sigma}}^{(o)}=\mathbf{C}_{o}^{s}\left(\mathbf{A}^{-1}-\mathbf{S}_{o}^{s}\right) & \\
& \times\left(\mathbf{A}^{-1}-\left(1-f_{1}\right) \mathbf{S}_{o}^{s}-f_{1} \mathbf{I}\right)^{-1} \mathbf{C}_{o}^{s^{-1}} d \overline{\boldsymbol{\sigma}} .
\end{aligned}
$$

Therefore, the total stress of the rubber matrix after a time increment is

$$
\boldsymbol{\sigma}^{(o)}(t+d t)=\boldsymbol{\sigma}^{(o)}(t)+d \boldsymbol{\sigma}^{(o)}(t)+d \overline{\boldsymbol{\sigma}}^{(o)}(t) .
$$

By using Eqs. (4.15) and (4.19), the effective stress, $\sigma_{e}^{(o)}(t+$ 


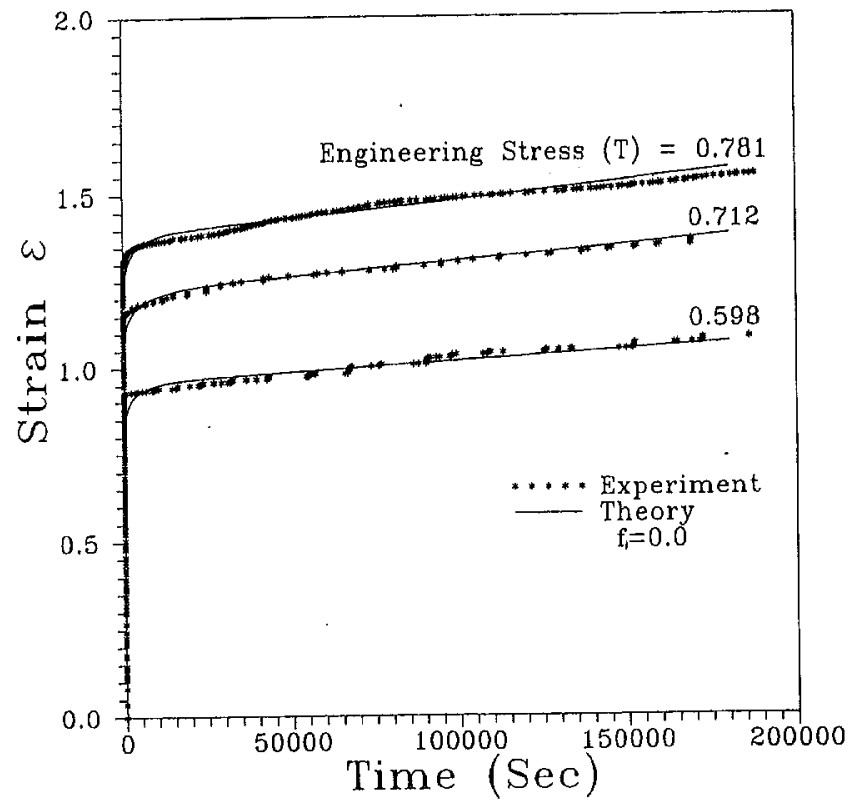

Fig. 2 The creep strain $\epsilon_{11}$ versus time curve of $f_{1}=0.00$

$d t)$, and $\lambda_{e}^{(o)}(t+d t)$ then can be derived. By substituting the results of $\sigma_{e}^{(o)}$ and $\lambda_{c}^{(o)}$ into Eqs. (2.31) and (2.38), we can have the strain increment of the rubber matrix $d \epsilon^{c}$. Once the result of $d \boldsymbol{\epsilon}^{c}$ is obtained, the procedures for deriving Eqs. (4.3)(4.19) will be repeated to get the quantities of $\overline{\boldsymbol{\epsilon}}(t), \boldsymbol{\sigma}^{(o)}(t)$ and $\boldsymbol{\epsilon}^{(o)}(t)$ for every time increment. Therefore, by using the small time-increment approach, we can predict the creep history of the solid-filled rubber composites.

\section{Experiments}

The basic elastomers were prepared from silicone rubber cured with the hardener T409103 (from Wacker-Chemie $\mathrm{GmbH}$ ). The material was cast into sheets measuring 150 by 150 by $2 \mathrm{~mm}$. The particulate composites were obtained by adding glass spheres of 66 to $88 \mathrm{~mm}$ in diameter in quantities necessary to obtain volume fractions of 13.3 percent and 21 percent in the silicone mixture. The Young's modulus of the glass beads was $68500 \mathrm{Mpa}$. The Poisson's ratio of the glass beads was 0.24 . The shear modulus of the silicone rubber $\mu$ was found to be $0.4 \mathrm{Mpa}$ by the aid of Eq. (2.8) and the method of least squares with the data obtained from simple tensile tests. Tensile and creep specimens of gauge lengths $20 \mathrm{~mm}$ (by ASTM D412) were cut from the sheets. All the specimens were kept in a desiccator for 15 days for post curing and to stabilize their properties. All the tensile and creep tests were performed in a laboratory environment of 50 percent relative humidity $(\mathrm{RH})$ and at $25^{\circ} \mathrm{C}$.

\section{Results and Discussions}

We assume in our calculations that the inclusions and the matrix take the properties of the glass beads and the silicone rubber, respectively, at room temperature. Based on two creep curves tested at $0.781 \mathrm{MPa}$ and $0.712 \mathrm{MPa}$, the creep properties of the silicone rubber at room temperature were found to be $\mu$ $=0.4 \mathrm{MPa}, \mu_{t}=3 \mathrm{MPa}, A_{t}=8.4 \times 10^{-6} 1 / \mathrm{sec}, \nu_{t}=7.831 /$ $\mathrm{MPa}$,

$$
A_{s}=5.2 \times 10^{-7} 1 / \mathrm{sec} \text { and } \nu_{s}=2.01 / \mathrm{MPa} .
$$

The quantitative accuracy of the present theory can to some extent be assessed by comparison between its predicted results and the experimental results. Such a comparison is shown in Figs. $2-4$. The volume fractions of 0 percent, 13.3 percent, and

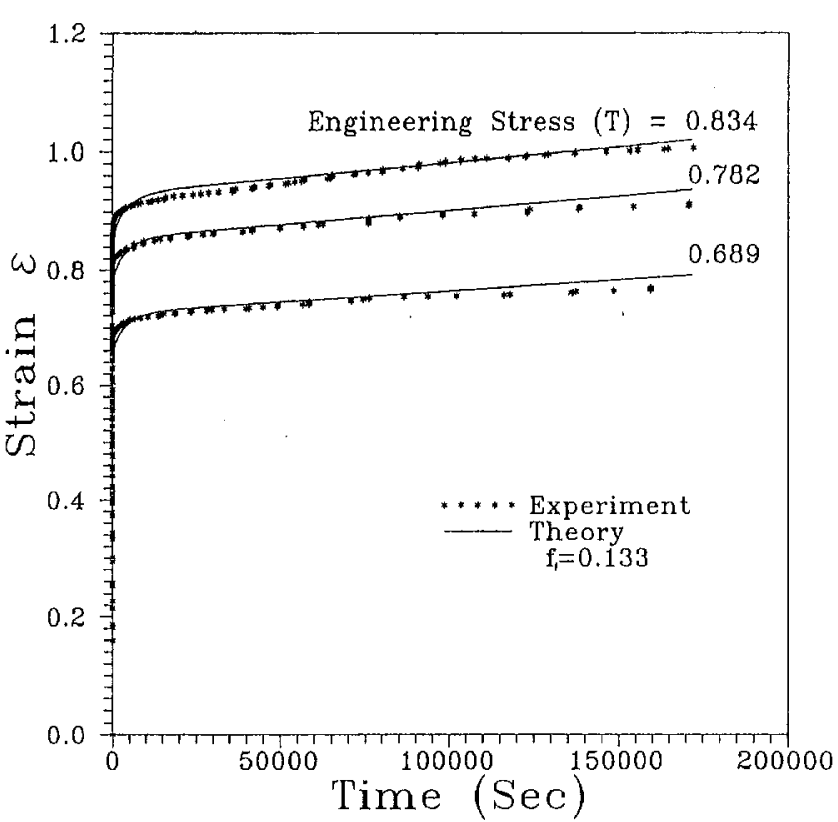

Fig. 3 The creep strain $\epsilon_{11}$ versus time curve of $f_{1}=0.13$

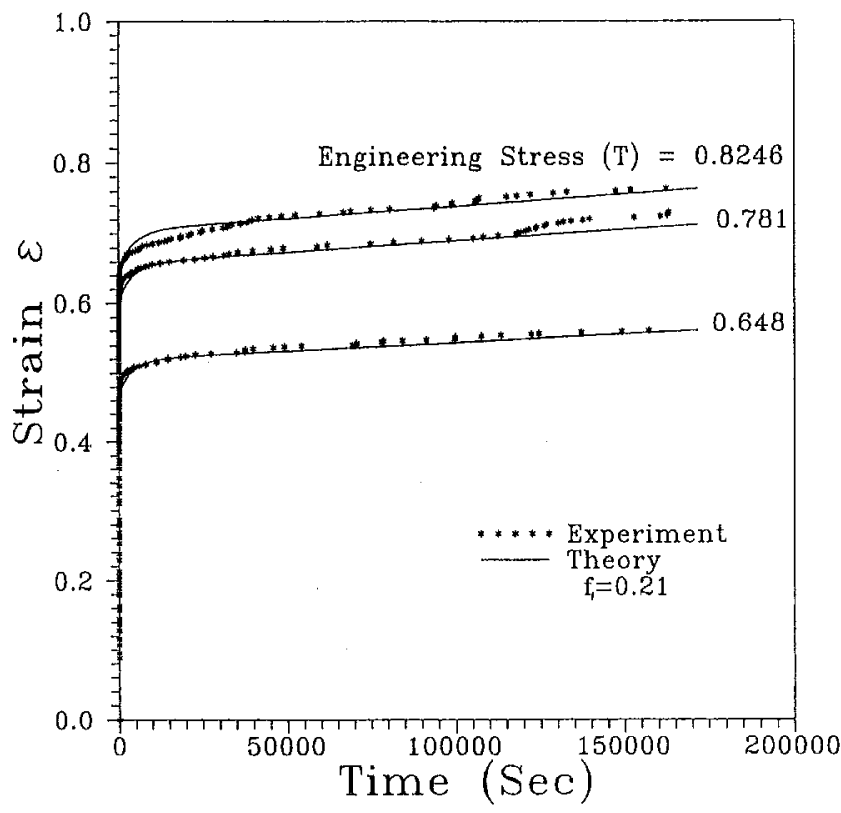

Fig. 4 The creep strain $\epsilon_{11}$ versus time curve of $f_{1}=0.21$

21 percent of glass bead-reinforced silicone rubber were under three different constant creep test loads, where the solid line represents the theoretical predictions, and the symbols represent the experimental results. The results show that filling of the glass beads had a reinforcing effect. The solution turns out to be in very good agreement with the experimental results.

\section{Acknowledgments}

The authors wish to express their thanks to Prof. Yih-Hsing Pao for his many helpful suggestions. This work was supported by the National Science Council of R.O.C. under grant NSC80-0405-E002-13.

\section{References}

Eshelby, J. D., 1957, "The Determination of the Elastic Field of an Ellipsoidal Inclusion and Related Problems," Proceedings of Royal Society, London, Vol. A241, pp. 376-396. 
Mori, T., and Tanaka, K., 1973, "Average Stress in Matrix and Average Elastic Energy of Materials with Misfitting Inclusions," Acta Metallurgica, Vol. 21, pp. $571-574$

Prandtl, L., 1925, "Spannungsverteilung in Plastichen Koerpern.," Proc. lst Internat. Congr. Appl. Mech., Deflt, pp. 43-54.

Reuss, E., 1930, "Beruecksichtigung der elastichen Formaenderungen in der Plasizitaetstheorie," Zeits. Angew. Math. Mech., Vol. 10, pp. 266-274.

So, H., and Chen, U. D., 1991, "A Nonlinear Mechanical Model for SolidFilled Rubbers,"' Polymer Engineering and Science, Vol. 31, pp. 410-416.

Tandon, G. P., and Weng, G. J., 1988, "A Theory of Particle-Reinforced Plasticity," ASME Journal OF APPLIED MECHANICS, Vol. 55, pp. 126135.

Truesdell, C., and Noll, W., 1965, "The non-linear field theories of mechanics," Encyclopedia of Physics, V. III/3, S. Flgge, ed., Springer-Verlag, Berlin.
Weng, G, J., 1990, "The overall elastoplastic stress-strain relation of dualphase metals," J. Mech. Phys. Solids, Vol. 38, pp. 419-441.

Weng, G. J., 1981, "Self-Consistent Determination of Time-Dependent Behavior of Metals," ASME JouRnal of APPLIED MEChanics, Vol. 48, pp. 41-46.

Zhao, Y. H., Tandon, G. P., and Weng, G. J., 1989, "Elastic Moduli for a Class of Porous Materials," Acta Mechanica, Vol. 76, pp. 105-130.

Zhu, Z. G., and Weng, G. J., 1987, "Micromechanics of Time-Dependent Deformation in a Dispersion Hardened Polycrystal," Acta Mechanica, Vol. 69, pp. $295-313$.

Zhu, Z. G., and Weng, G. J., 1990, "Creep Anisotropy of MetalMatrix Composite Containing Dilute Concentration of Aligned Spheroidal Inclusions," Mechanics of Materials, Vol. 9, pp. 93-105.

Zhu, Z. G., and Weng, G. J., 1989, "Creep Deformation of Particle Strengthened Metal Matrix Composites," ASME Journal of Engineering Materials and Technology, Vol. 111, pp. 99-105. 